Supporting Information (SI)

\title{
Enhancement of the Thermoelectric Performance of 2D SnSe Nanoplates through Incorporation of Magnetic Nanoprecipitates
}

\author{
Sushmita Chandra, Prabir Dutta and Kanishka Biswas* \\ New Chemistry Unit, International Centre for Material Science and School of Advanced \\ Materials, Jawaharlal Nehru Centre for Advanced Scientific Research (JNCASR), \\ Jakkur P.O., Bangalore 560064, India \\ *Email: kanishka@jncasr.ac.in
}

\section{DETAILED EXPERIMENTAL SECTION}

Reagents. Tin (II) chloride dihydrate $\left(\mathrm{SnCl}_{2} \cdot 2 \mathrm{H}_{2} \mathrm{O}, 98 \%\right.$, Sigma Aldrich), selenium powder (Se, $99.99 \%$, Alfa Aesar), gadolinium (III) acetate hydrate $\left(\mathrm{Gd}\left(\mathrm{OOCCH}_{3}\right)_{3} \cdot \mathrm{xH}_{2} \mathrm{O}, 99.9 \%\right.$, Alfa Aesar) and sodium hydroxide Pellets ( $\mathrm{NaOH}, 98 \%$ Sigma Aldrich) were used for the synthesis.

Synthesis of $\mathrm{Sn}_{1-\mathrm{x}} \mathbf{G d}_{\mathrm{x}} \mathrm{Se}$ nanoplates. $\mathrm{Sn}_{1-\mathrm{x}} \mathrm{Gd}_{\mathrm{x}} \mathrm{Se}(\mathrm{x}=$ 0-3 mol\%) nanoplates were synthesized using simple hydrothermal method. First a mixture of $\mathrm{SnCl}_{2} \cdot 2 \mathrm{H}_{2} \mathrm{O}(2 \mathrm{mmol})$ and $\mathrm{Gd}\left(\mathrm{OOCCH}_{3}\right)_{3} \cdot \mathrm{xH}_{2} \mathrm{O}\left(\mathrm{x} \mathrm{mmol}^{2}\right)$ were dissolved in deionized water. The solution was then sonicated at room temperature for $10 \mathrm{~min}$, followed by addition of $\mathrm{NaOH}(30 \mathrm{mmol})$ and sonicated again for another 10 min unless a clear solution has been obtained. The mixture was then transferred to a Teflon-lined stainless-steel autoclave of $25 \mathrm{ml}$ capacity and Se powder (1 mmol) was added to it. The autoclaves were then sealed tightly and heated to $130{ }^{\circ} \mathrm{C}$ for 36 hrs. After cooling to room temperature, the black precipitate was collected and thoroughly washed with absolute ethanol and deionized water for several times. Finally, the product was vacuum dried at $60{ }^{\circ} \mathrm{C}$ for $6 \mathrm{hrs}$ and collected as powder. During the synthesis, $\mathrm{NaOH}$ acts as a solubilizing agent. Excess of $\mathrm{Sn}^{2+}$ should always be present in the reaction mixture, as $\mathrm{Sn}^{2+}$ itself acts as a reductant and precipitant. The yield of the reaction was $\sim 95 \%$ and the nanoplates 
were scaled up to $\sim 10 \mathrm{gm}$ for the thermoelectric measurements. The nanoplates were dispersed in ethanol for further characterizations.

Spark plasma sintering (SPS). SPS was done using a SPS211-LX (Dr. Sinter Lab) instrument. The powdered nanoplates were sintered to prepare a cylinder $(10 \mathrm{~mm} \times 10 \mathrm{~mm})$ using graphite dies at $50 \mathrm{MPa}$ pressure and $450{ }^{\circ} \mathrm{C}$ temperature. The samples are cut and polished in different directions to measure the anisotropic electrical and thermal transport properties of SnSe. The density of the SPS processed samples were found to be about $\sim 95 \%$ of theoretical density.

Powder X-ray diffraction. Powder X-ray diffraction for all the samples were recorded using a $\mathrm{Cu} \mathrm{K}_{\alpha}(\lambda=1.5406 \AA)$ radiation on a Bruker D8 diffractometer.

Field emission scanning electron microscopy (FESEM). FESEM imaging and EDAX were performed using NOVA NANO SEM 600 (FEI, Germany) operated at $15 \mathrm{kV}$.

Transmission electron microscopy (TEM). TEM experiments were performed using JEOL JEM3010 TEM fitted with a Gatan CCD camera operating at $300 \mathrm{kV}$ accelerating voltage. A suspension of the nanosheets was prepared in cyclohexane solution and it was then drop casted in a holey carbon coated $\mathrm{Cu}$ grid for TEM imaging.

Scanning transmission electron microscopy (STEM). STEM imaging was carried out using FEITECNAI G 20 STWIN TEM operating at $200 \mathrm{kV}$. The sample preparation was same as that of TEM. EDAX compositional analysis and color mapping were performed during STEM imaging. Background was subtracted (using multi-polynomial model) during the data processing for EDAX color mapping (with $500 \mathrm{eV}$ minimum region of interest width). Errors in the determination in compositions of nanosheets in EDAX measurements is nearly $5 \%$.

Inductively coupled plasma atomic emission spectroscopy (ICP-AES). The exact composition of the as synthesized nanosheets was calculated based on ICP-AES data. ICP- 
AES measurements were carried out using Perkin-Elmer Optima 7000DV instrument. ICPAES measurement were carried out by dissolving the powder nanosheets in aqua regia $\left(\mathrm{HNO}_{3}: \mathrm{HCl}=1: 3\right)$ followed by diluting with millipore water. Sn standard $(1000 \mathrm{mg} / \mathrm{L}$, SigmaAldrich), Se standard (1000 mg/L, Sigma-Aldrich) and Gd standard (1000 mg/L, SigmaAldrich) were used to determine the compositions in ICP. In the present measurement, error bar lies below $1.5 \%$.

Magnetic measurements. The dc magnetic measurements were performed using a vibrating sample magnetometer from Cryogenic Ltd., U.K. Temperature dependent magnetization $(M)$ data were recorded in two different protocols namely zero field cooling (ZFC) and field cooling (FC). In ZFC mode, the sample was first cooled from room temperature to $2 \mathrm{~K}$ without any magnetic field $(H)$.Then a magnetic field of $1 \mathrm{kOe}$ is applied and subsequently recorded the $M$ data during heating from $2 \mathrm{~K}$ to $300 \mathrm{~K}$. Whereas in FC mode, the sample was first cooled in presence of $H=1 \mathrm{kOe}$ and then measured the $M$ during subsequent heating. $M-H$ curves at different constant temperature were recorded in the $\pm 150 \mathrm{kOe}$ magnetic field range.

Electrical transport properties. Electrical conductivity $(\sigma)$ and Seebeck coefficients $(S)$ were measured simultaneously from the rectangular bar under helium atmosphere from room temperature to $873 \mathrm{~K}$ using a ULVAC-RIKO ZEM-3 instrument system. $\sigma$ and $S$ were measured in both the parallel and perpendicular to the pressing directions. Electrical and thermal transports are measured in same direction.

Hall measurement. Room temperature carrier concentrations were determined from Hall coefficient measurements with the equipment developed by Excel Instrument, India. Fourcontact Hall-bar geometry was used for the measurement. The maximum applied magnetic field was 1 Tesla. Room temperature carrier concentration, $n$, was measured using the formula $n=1 / e R_{H}$, where $e$ is the electronic charge, $R_{H}$ is hall coefficient. 
Thermal Transport. The thermal diffusivity $(D)$ of the coin-shaped samples have been measured by laser flash diffusivity technique using NETZSCH LFA 457 instrument in 300-873 K range. Total thermal conductivity $\left(\kappa_{t o t}\right)$ was calculated using the formula $\kappa_{t o t}=$ $D C_{p} \rho$, where $D$ is the thermal diffusivity, $C_{p}$ is specific heat, and $\rho$ is density of the sample. $\mathrm{C}_{p}$ was derived experimentally using pyroceram as a reference material during the thermal diffusivity measurement. Further, the electronic thermal conductivities, $\kappa_{\text {ele }}$ were estimated using Wiedemann-Franz Law, $\kappa_{e l e}=L \sigma T$, where $L$ is the Lorenz number which is estimated by fitting reduced chemical potential derived from temperature-dependent Seebeck coefficient using single parabolic band conduction and dominant acoustic phonon scattering of carriers. 


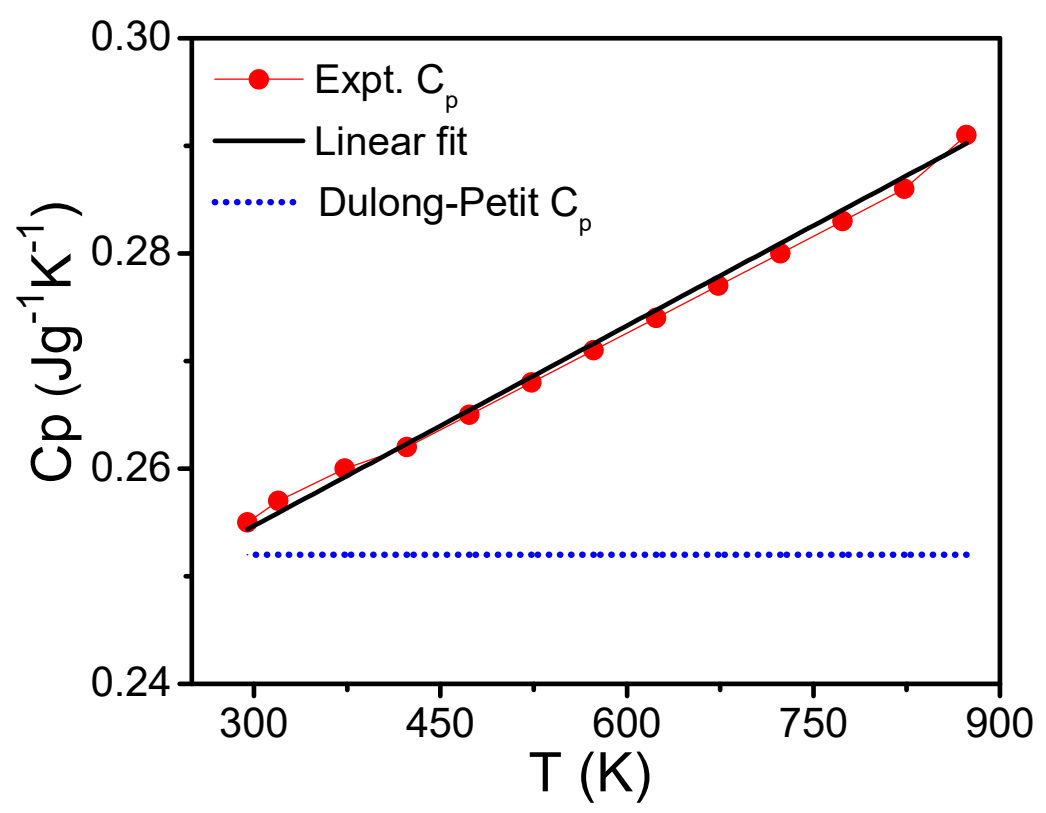

Figure S1. Heat capacity $\left(C_{p}\right)$ of the as-synthesized SnSe nanoplates along with the DulongPetit $C_{p}$ value.

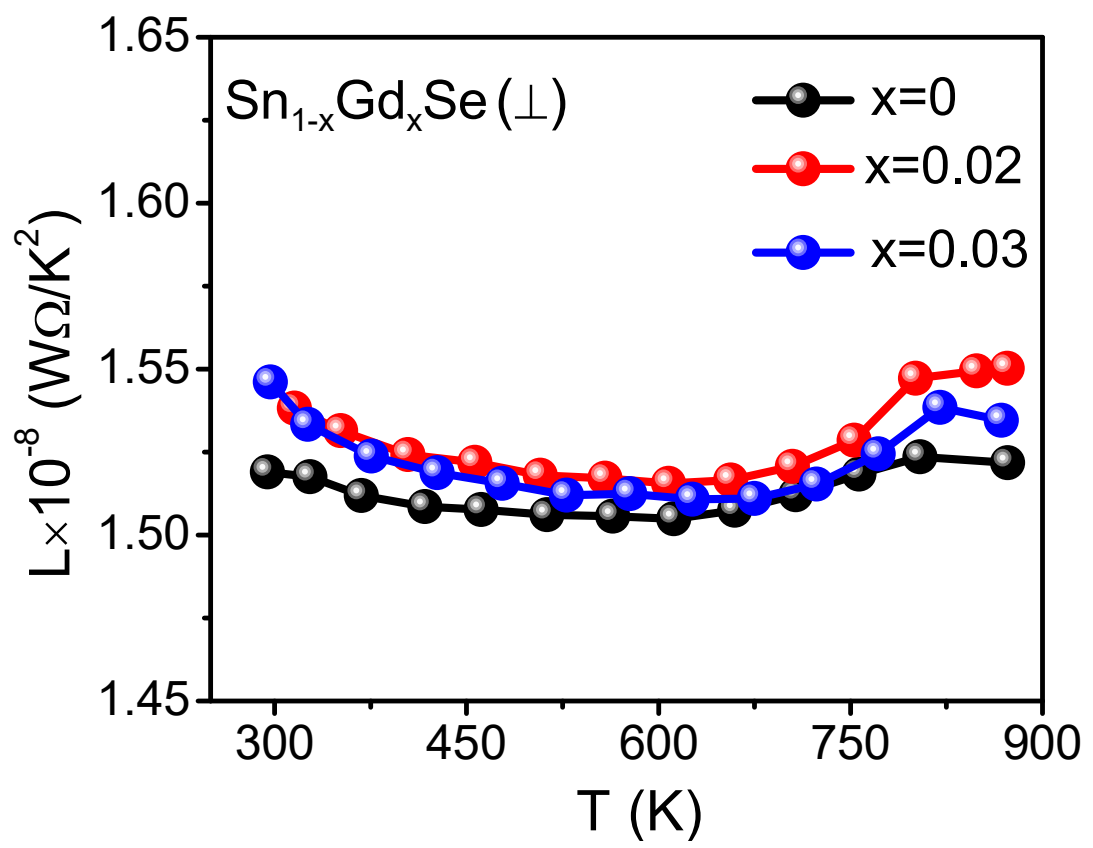

Figure S2. Lorenz number of SPS processed $\mathrm{Sn}_{0.97} \mathrm{Gd}_{0.03}$ Se nanoplates obtained by fitting the reduced chemical potential. 


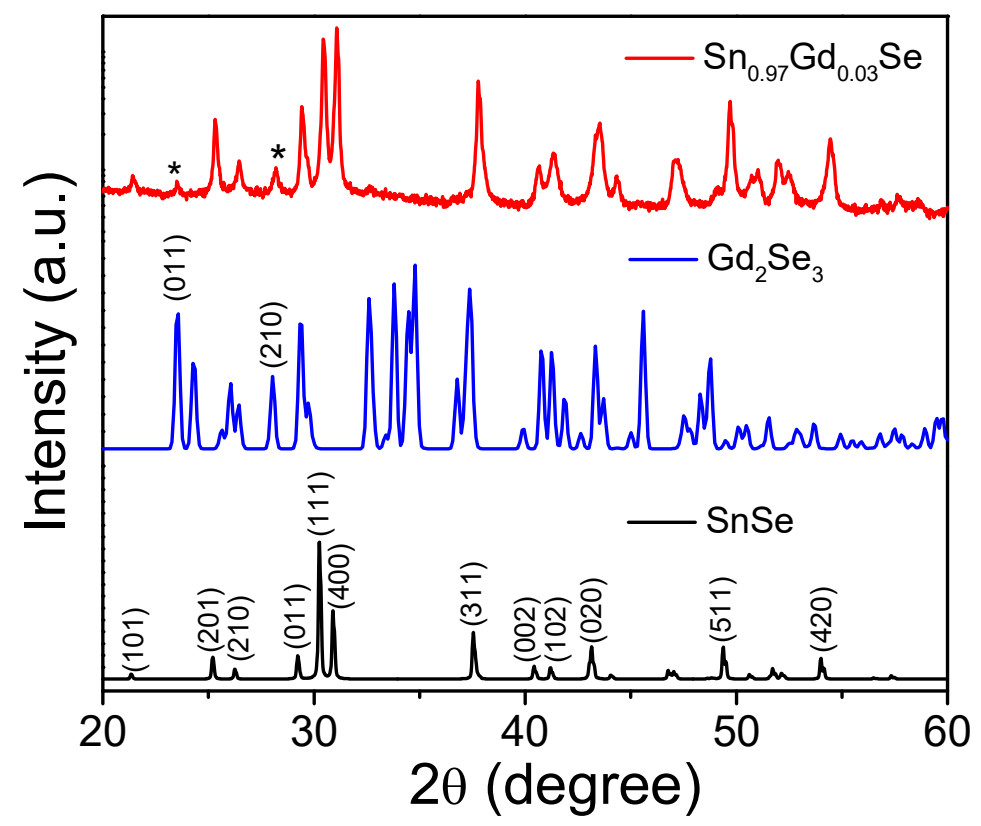

Figure S3. PXRD pattern of the $\mathrm{Sn}_{0.97} \mathrm{Gd}_{0.03} \mathrm{Se}$ nanoplates indicating the presence of $\mathrm{Gd}_{2} \mathrm{Se}_{3}$ secondary phase in SnSe matrix.
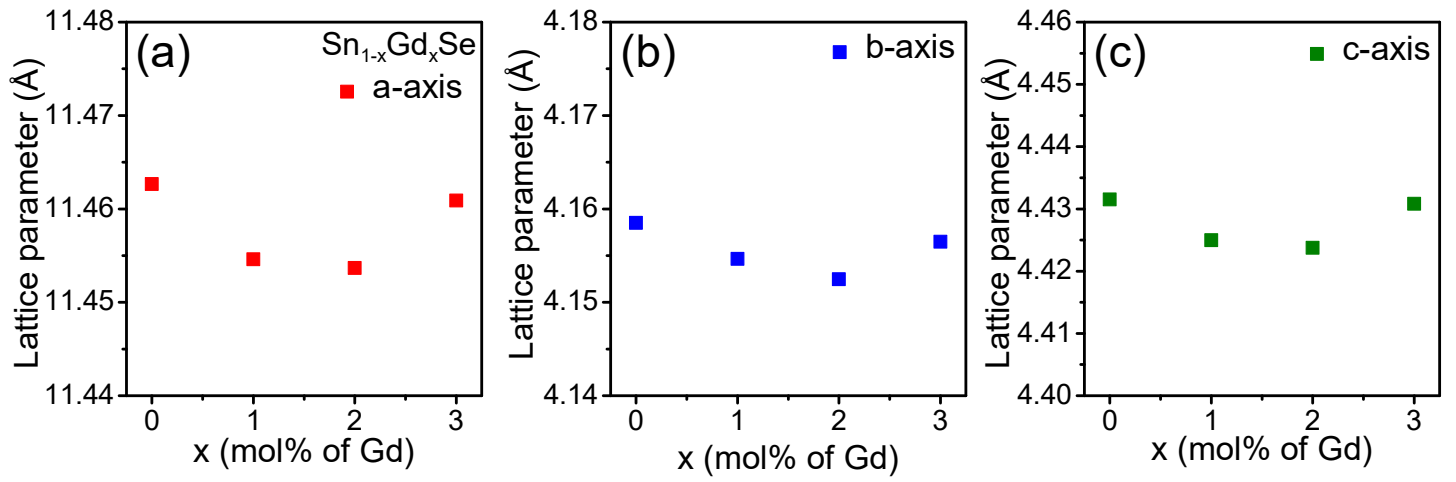

Figure S4. Lattice parameters of the $\mathrm{Sn}_{1-\mathrm{x}} \mathrm{Gd}_{\mathrm{x}} \mathrm{Se}(\mathrm{x}=0-3 \mathrm{~mol} \%)$ nanoplates derived from the PXRD. 

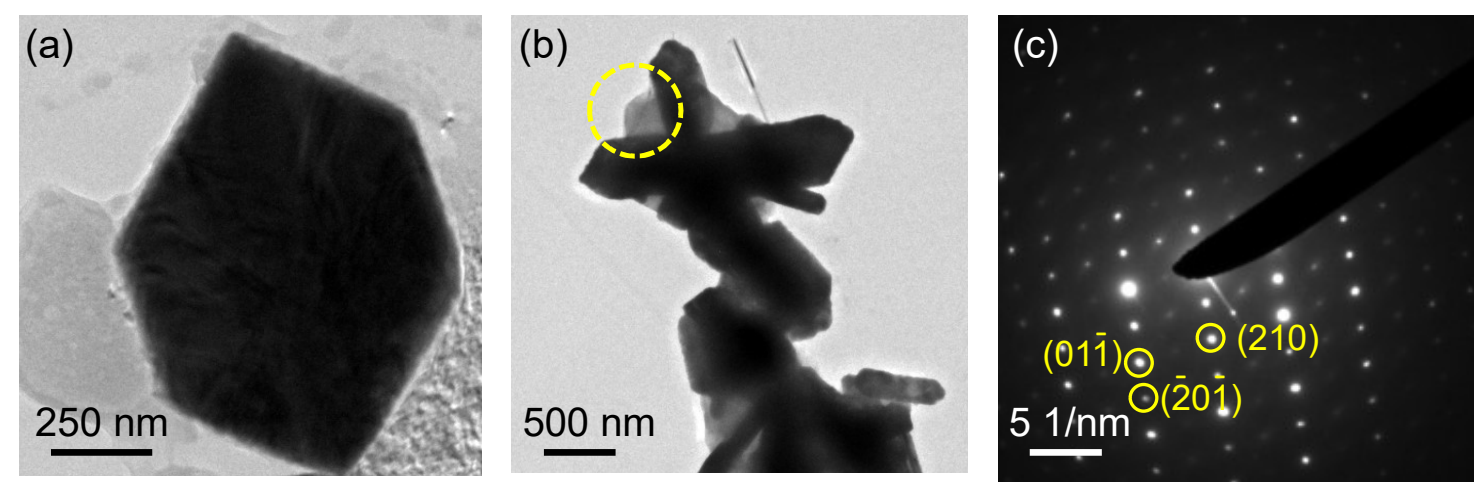

Figure S5. (a) and (b) TEM images and (c) SAED pattern of pristine SnSe nanoplates

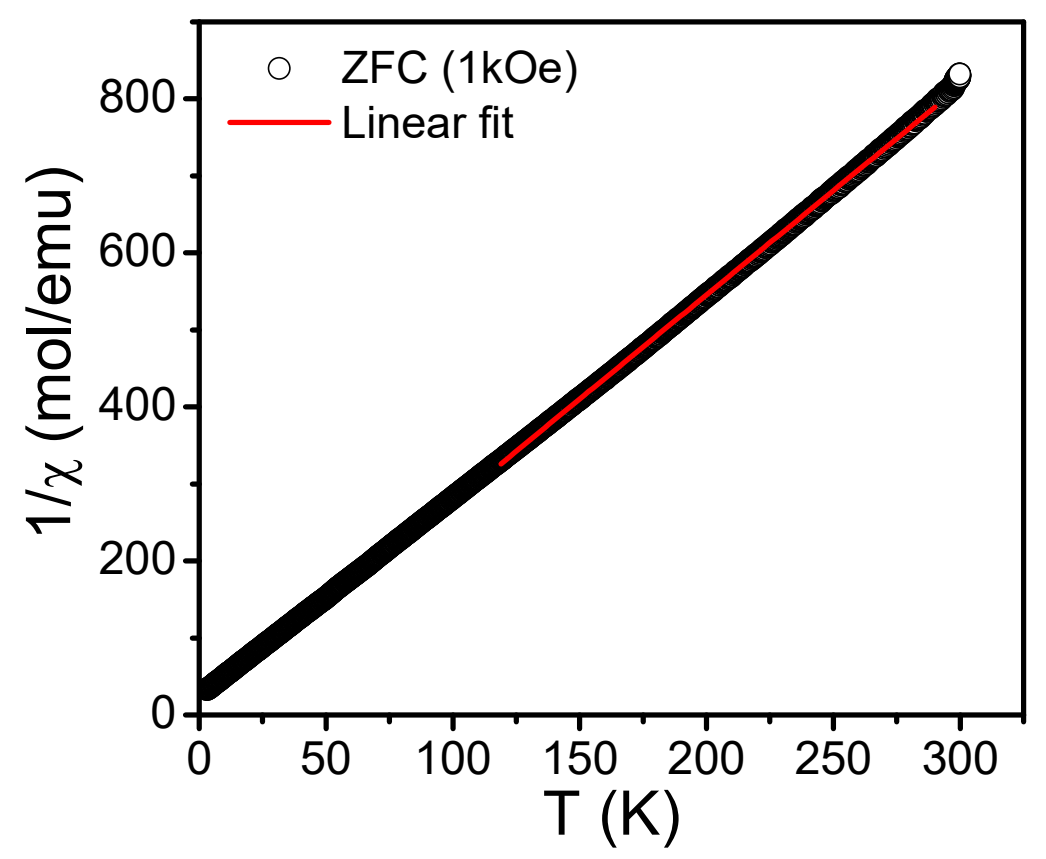

Figure S6. Temperature dependent magnetic susceptibility $(\chi)$ of $\mathrm{Sn}_{0.97} \mathrm{Gd}_{0.03} \mathrm{Se}$ nanoplates which obeys the Curie-Weiss law in the temperature range of 100-300 K. 


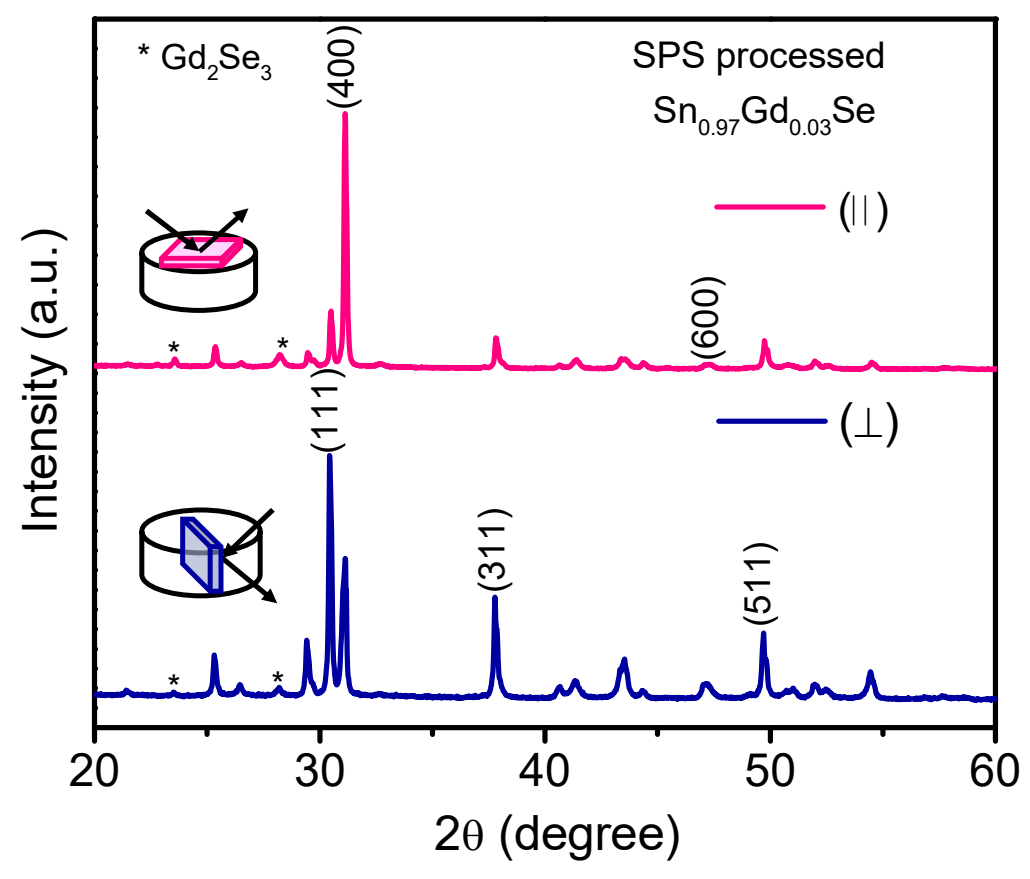

Figure S7. PXRD patterns of SPS processed $3 \mathrm{~mol} \%$ Gd-doped SnSe along the parallel and perpendicular directions revealing the anisotropic nature of the sample. The direction of X-ray diffraction has been denoted by the schematic diagrams in inset.
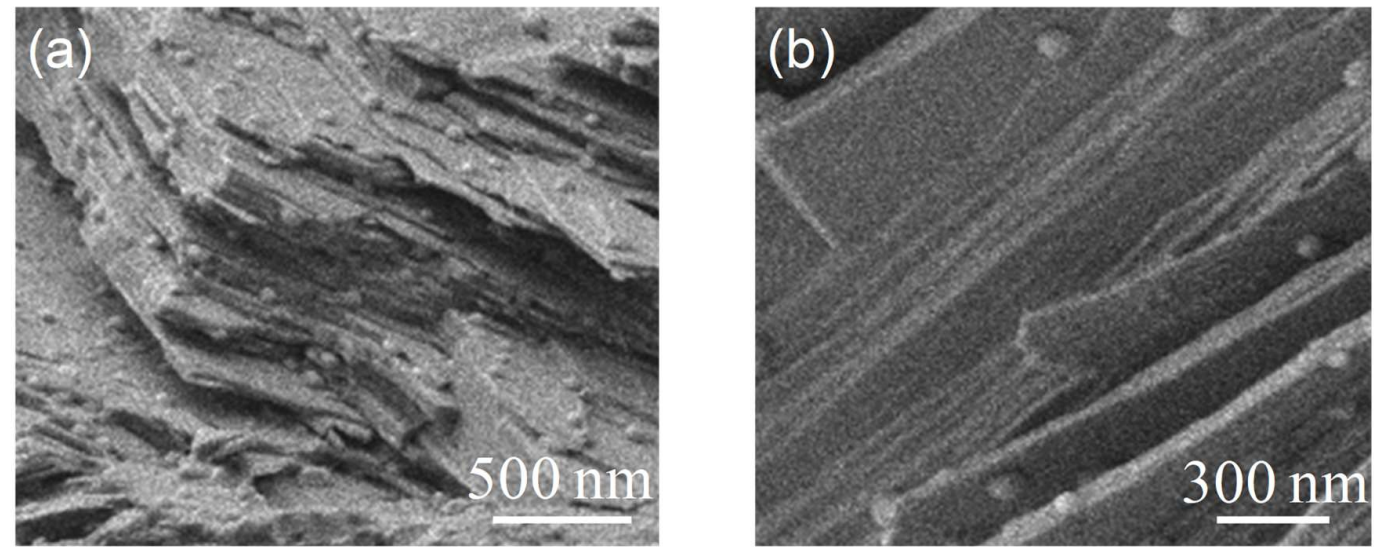

Figure S8. (a) and (b) FESEM images taken from the fractured surface of SPS processed $\mathrm{Sn}_{0.97} \mathrm{Gd}_{0.03} \mathrm{Se}$ nanoplates showing the retention of the layered morphology. 
(a)

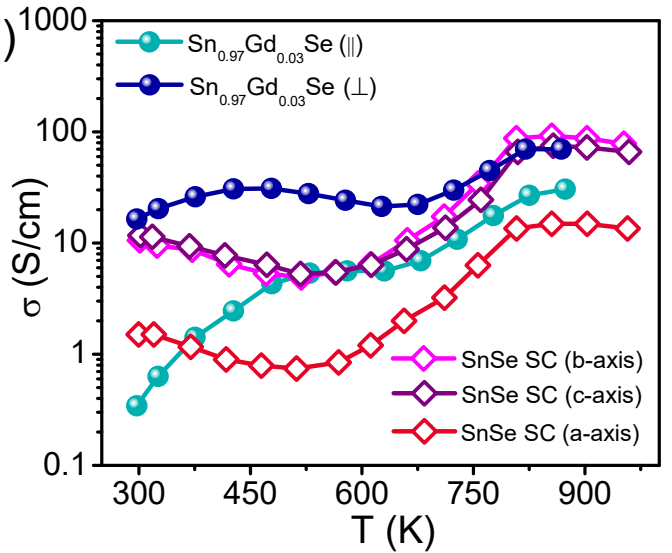

(c)

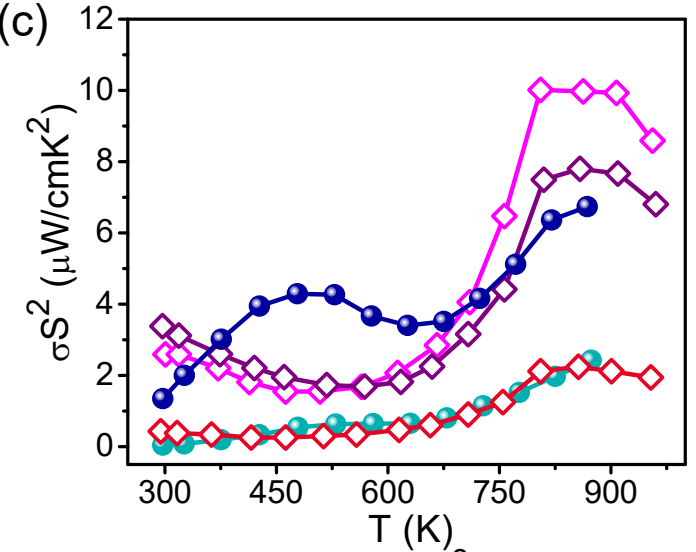

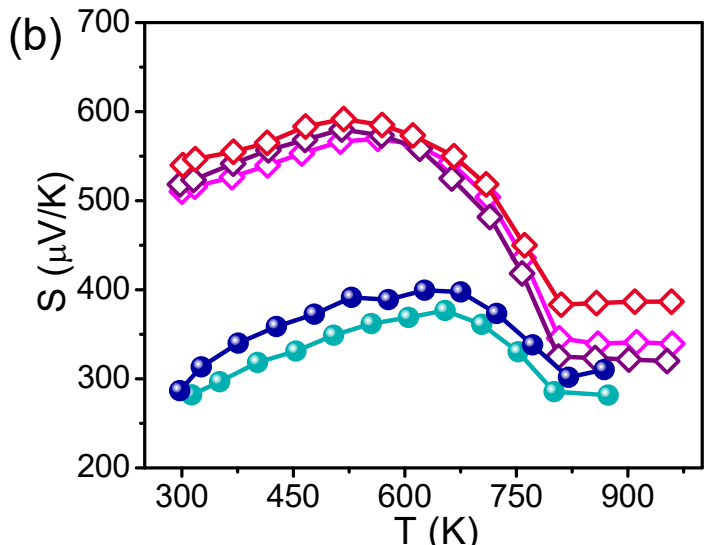

(d)

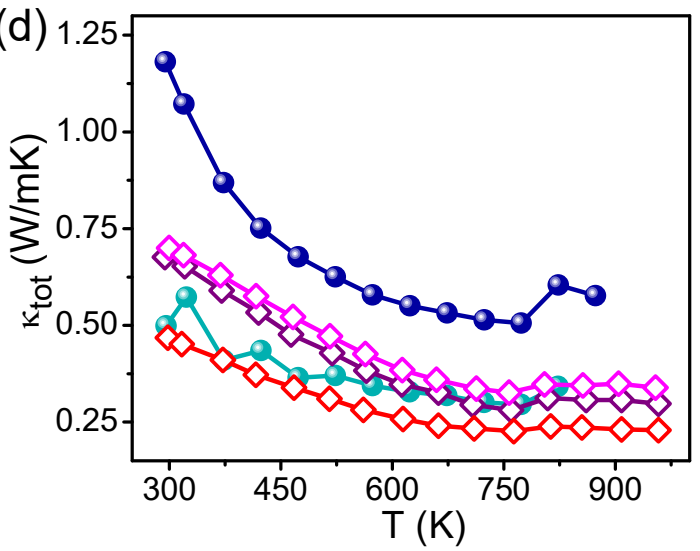

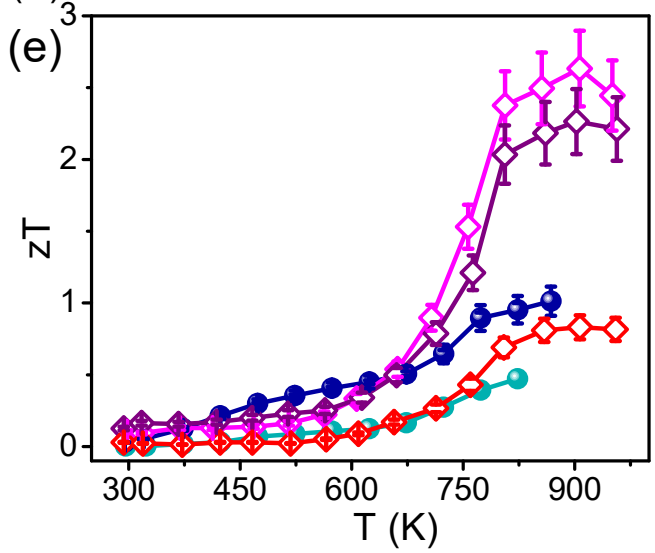

Figure S9. Anisotropic (a) $\sigma$, (b) $S$, (c) $S^{2} \sigma$, (d) $\kappa_{t o t}$ and (e) $z T$ of SPS processed $\mathrm{Sn}_{0.97} \mathrm{Gd}_{0.03} \mathrm{Se}$ nanoplates measured along parallel and perpendicular to SPS pressing directions. Single crystals (SC) data of SnSe (from Nature 2014, 508, 373-377) has been also provided for comparison. 


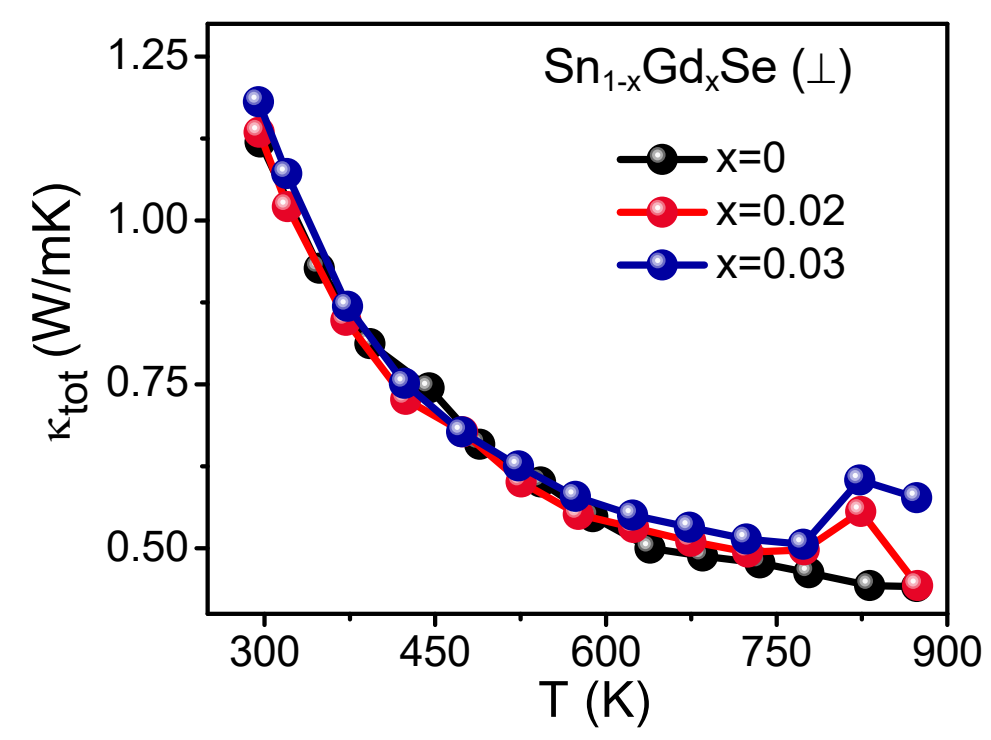

Figure S10. Temperature dependent total thermal conductivity of $\operatorname{Sn}_{1-\mathrm{x}} \mathrm{Gd}_{\mathrm{x}} \operatorname{Se}$ nanoplates $(\mathrm{x}=$ $0,2,3 \mathrm{~mol} \%)$.
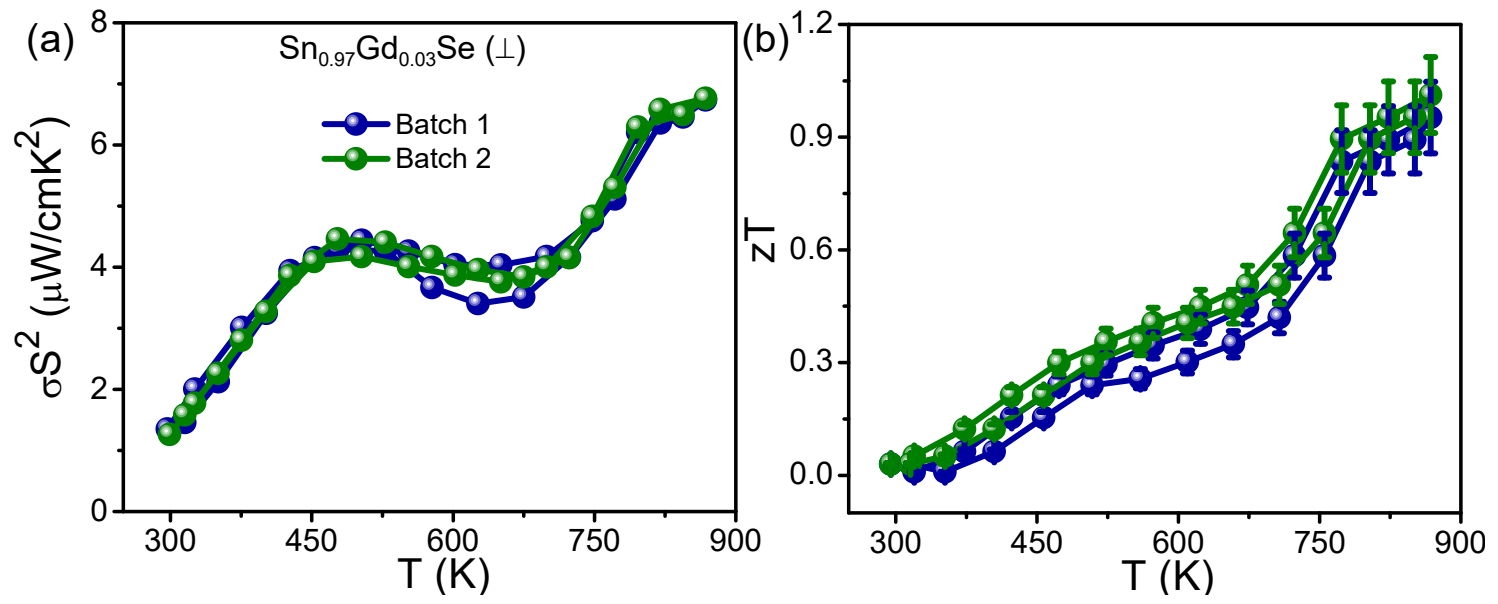

Figure S11. The reversibility and reproducibility of the thermoelectric data of the SPS processed $\mathrm{Sn}_{0.97} \mathrm{Gd}_{0.03} \mathrm{Se}$ nanoplates are evident from the temperature dependent (a) power factor and (b) $z T$ plots measured for different batches (synthesized separately) with the heating cooling cycles. 
Table S1. Density of the $\mathrm{Sn}_{1-\mathrm{x}} \mathrm{Gd}_{\mathrm{x}} \mathrm{Se}$ nanoplates.

\begin{tabular}{|c|c|}
\hline Composition & Density $\left(\mathbf{g} / \mathbf{c m}^{\mathbf{3}}\right)$ \\
\hline $\mathrm{SnSe}$ & 5.78 \\
\hline $\mathrm{Sn}_{0.99} \mathrm{Gd}_{0.01} \mathrm{Se}$ & 5.87 \\
\hline $\mathrm{Sn}_{0.98} \mathrm{Gd}_{0.02} \mathrm{Se}$ & 5.81 \\
\hline $\mathrm{Sn}_{0.97} \mathrm{Gd}_{0.03} \mathrm{Se}$ & 5.88 \\
\hline
\end{tabular}

Table S2. Charge carrier concentration and mobility of the $\mathrm{Sn}_{1-\mathrm{x}} \mathrm{Gd}_{\mathrm{x}} \mathrm{Se}$ nanoplates.

\begin{tabular}{|c|c|c|}
\hline Composition & $\begin{array}{c}\text { Carrier concentration } \\
\mathbf{n}\left(\mathbf{c m}^{-\mathbf{3}} \mathbf{)}\right.\end{array}$ & $\begin{array}{c}\text { Carrier mobility } \\
\mu\left(\mathbf{c m}^{\mathbf{2}} \mathbf{V}^{\mathbf{- 1}} \mathbf{s}^{\mathbf{1}} \mathbf{)}\right.\end{array}$ \\
\hline $\mathrm{SnSe}$ & $3.9 \times 10^{17}$ & 150.9 \\
\hline $\mathrm{Sn}_{0.98} \mathrm{Gd}_{0.02} \mathrm{Se}$ & $2.6 \times 10^{17}$ & 221.6 \\
\hline $\mathrm{Sn}_{0.97} \mathrm{Gd}_{0.03} \mathrm{Se}$ & $3.1 \times 10^{19}$ & 3.4 \\
\hline
\end{tabular}

Table S3. Measured sample compositions from ICP-AES and EDAX.

\begin{tabular}{|c|c|c|}
\hline $\begin{array}{c}\text { Nominal } \\
\text { composition }\end{array}$ & $\begin{array}{c}\text { Composition obtained } \\
\text { from ICP-AES }\end{array}$ & $\begin{array}{c}\text { Composition obtained } \\
\text { from EDAX }\end{array}$ \\
\hline $\mathrm{SnSe}$ & $\mathrm{SnSe}_{1.03}$ & $\mathrm{SnSe}_{0.99}$ \\
\hline $\mathrm{Sn}_{0.99} \mathrm{Gd}_{0.01} \mathrm{Se}$ & $\mathrm{Sn}_{0.98} \mathrm{Gd}_{0.007} \mathrm{Se}_{1.11}$ & - \\
\hline $\mathrm{Sn}_{0.98} \mathrm{Gd}_{0.02} \mathrm{Se}$ & $\mathrm{Sn}_{0.987} \mathrm{Gd}_{0.023} \mathrm{Se}_{1.19}$ & - \\
\hline $\mathrm{Sn}_{0.97} \mathrm{Gd}_{0.03} \mathrm{Se}$ & $\mathrm{Sn}_{0.969} \mathrm{Gd}_{0.035} \mathrm{Se}_{1.01}$ & $\mathrm{Sn}_{0.953} \mathrm{Gd}_{0.031} \mathrm{Se}_{1.31}$ \\
\hline
\end{tabular}

